\title{
Limiting current in a crossed-field gap
}

Y. Y. Lau

Intense Energy Beam Interaction Laboratory and Department of Nuclear Engineering, University of Michigan, Ann Arbor, Michigan 48109-2104 and Applied Physics Program. University of Michigan, Ann Arbor, Michigan 48109

P. J. Christenson

Intense Energy Beam Interaction Laboratory and Department of Nuclear Engineering, University of Michigan, Ann Arbor, Michigan 48109-2104

David Chernin

Science Applications International Corporation, 1710 Goodridge Drive, Mclean, Virginia 22102

(Received 18 June 1993; accepted 7 September 1993)

An analytic theory is presented that yields the maximum transmittable current across an anodecathode gap that is embedded in an arbitrary transverse magnetic field $(B)$. The limiting current is found to be relatively insensitive to $B$ for all $B<B_{\mathrm{H}}$, where $B_{\mathrm{H}}$ is the Hull cutoff magnetic field required for magnetic insulation. The classical Child-Langmuir solution is recovered in the limit $B \rightarrow 0$.

\section{INTRODUCTION}

There are two mechanisms that limit the electron flow across an anode-cathode gap. The first is the self-potential associated with the electrons' space charge within the gap. When the emitted current is too high, this self-potential is sufficient to prohibit beam propagation. The simplest form of this limiting current is the classical Child-Langmuir law. ${ }^{1}$ Because of its fundamental importance in electroncircuit interaction, ${ }^{2}$ the Child-Langmuir law has been extended to the relativistic regime, ${ }^{3}$ to a time-varying gap voltage, ${ }^{4}$ and to nanostructures, ${ }^{5}$ where quantum effects become important.

The second mechanism arises when there is an external magnetic field, $B$, parallel to the cathode surface. ${ }^{6,7}$ Under the direct current $(\mathrm{dc})$ condition, an electron emitted from the cathode with initial velocity $u_{0}$ would not be able to reach the anode if this transverse magnetic field $B>B_{\mathrm{H}}$, regardless of the amount of space charge within the gap. Here,

$$
B_{\mathrm{H}}=\sqrt{\frac{2 m V}{e D^{2}}+\left(\frac{m u_{0}}{e D}\right)^{2}}
$$

is the Hull cutoff magnetic field, ${ }^{6}$ expressed in terms of the anode voltage $V$, gap separation $D$, electron charge $e$ and electron mass $m$. Since $B_{\mathrm{H}}$ is independent of the emission current, it is derived from single particle orbit theory.

In this paper, we generalize the classical ChildLangmuir law to include the effect of an arbitrary transverse magnetic field $B$ and of an arbitrary initial velocity $u_{0}$. While some of the equations and their solutions are well known, ${ }^{8-11}$ we have gone a step further. We present a previously unknown analytic expression for the limiting current and, perhaps also for the first time, link this limiting current to the Hull cutoff. We show that the limiting current is relatively insensitive to $B$ for all $B<B_{\mathrm{H}}$, especially for the $u_{0} \cong 0$ case. For $B>B_{\mathrm{H}}$, the electrons fail to reach the anode, as expected.
In Sec. II, we present the analytic theory, and in Sec. III, we discuss some of the implications.

\section{ANALYSIS}

Consider a time-independent, one-dimensional, nonrelativistic model. In the Cartesian coordinates, the cathode is located at the plane $x=0$ and the anode at $x=D$. An external magnetic field $\hat{z} B$ is imposed and the anode is held at a voltage $V$ with respect to the cathode. We assume that all electrons are emitted with the same initial velocity $\hat{x} u_{0}$, carrying a current density $\hat{x} J$. We shall calculate the maximum value of $J$ (in terms of $V, B, D, u_{0}$ ) if this monoenergetic beam is to reach the anode. Since we are considering only a monoenergetic beam, the electrons will either all reach the anode or they will all return to the cathode. Thus it suffices to consider the orbit of a representative electron under the combined action of the external $B$ field and the self-consistent electrostatic potential $\phi(x)$.

Let $(u, v, 0)$ be the velocity of an electron and $n$ be the electron number density. From conservation of energy,

$$
e \phi(x)=\frac{1}{2} m\left(u^{2}+\Omega^{2} x^{2}\right)-\frac{1}{2} m u_{0}^{2}
$$

we may write the Poisson equation

$$
\frac{d^{2} \phi}{d x^{2}}=\frac{e n}{\epsilon_{0}}=\frac{J}{\epsilon_{0} u}
$$

in the following form:

$$
u \frac{d}{d x}\left(u \frac{d u}{d x}\right)+\Omega^{2} u=\frac{e J}{m \epsilon_{0}},
$$

where $\Omega=e B / m$ is the cyclotron frequency. We have used the relation $v=\Omega x$ in Eq. (2) and the continuity equation $J=e n u=$ constant in Eq. (3).

Equation (4) may be cast in the time domain with a change of variable $t=\int d x / u$. It then becomes a secondorder ordinary differential equation with constant coefficients which is simply the crossed-field analog of Llewellyn's equation. ${ }^{8}$ This equation may easily be solved 
for $u(t)$. In terms of nondimensional variables $\tilde{x}=x / D$, $\tilde{t}=\Omega t, J=e J / m \epsilon_{0} \Omega^{3} D, \widetilde{V}=e V / m D^{2} \Omega^{2}, \widetilde{u}_{0}=u_{0} / \Omega D$, this solution reads

$$
\tilde{u}(\tilde{t})=\widetilde{J}+\left(\tilde{u}_{0}-\tilde{J}\right) \cos (\tilde{t})+\tilde{c} \sin (\tilde{t})
$$

which may be integrated to yield ${ }^{8}$

$$
\tilde{x}(\tilde{t})=\widetilde{J} \tilde{t}+\left(\tilde{u}_{0}-\widetilde{J}\right) \sin (\tilde{t})+\widetilde{c}[1-\cos (\tilde{t})] .
$$

In Eqs. (5) and (6), $\tilde{c}$ represents the initial acceleration of the electron, it is proportional to the electric field on the cathode surface. With the use of Eq. (5), $\tilde{c}$ may be expressed in terms of the transit time $\widetilde{T}$ and the final velocity $\tilde{u}_{f}$ with which the electron reaches the anode. Upon substituting this expression of $\tilde{c}$ into Eq. (6), Eq. (6) becomes

$$
\tilde{J}=\frac{1-\left(\tilde{u}_{0}+\tilde{u}_{f}\right) \tan (\tilde{T} / 2)}{\widetilde{T}-2 \tan (\tilde{T} / 2)}
$$

when evaluated at $\tilde{t}=\tilde{T}$, at which $\tilde{x}(\tilde{T})=1$. From Eqs. (1) and (2), we obtain the useful relationships

$$
\begin{aligned}
& \tilde{u}_{f}=\left(\tilde{u}_{0}^{2}+2 \widetilde{V}-1\right)^{1 / 2}=\left[\left(B_{\mathrm{H}} / B\right)^{2}-1\right]^{1 / 2}, \\
& \left(B_{\mathrm{H}} / B\right)^{2}=2 \widetilde{V}+\widetilde{u}_{0}^{2} .
\end{aligned}
$$

The limiting current is determined when Eq. (7) admits no meaningful solution of $\widetilde{T}$ (i.e., when such solutions imply either $x<0$ or $d x / d t<0$ ). It is straightforward to show from Eq. (7) that this condition reads $\widetilde{J}_{\widetilde{J}}=\widetilde{J}_{c}$, where the limiting current $\widetilde{J}_{c}$ is determined from

$$
\tilde{J}_{c}=\frac{1}{\widetilde{T}-\sin (\widetilde{T})}
$$

and $\widetilde{T}(0<\tilde{T}<2 \pi)$ is the solution of

$$
\tilde{u}_{f}=-\tilde{u}_{0}+\frac{2 \sin ^{2}(\tilde{T} / 2)}{\tilde{T}-\sin (\tilde{T})} .
$$

Before we proceed further, let us show that Eq. (10) indeed reduces to the classical result as $\Omega \rightarrow 0$. In this limit, $\widetilde{T} \rightarrow 0, \widetilde{u}_{f} \rightarrow\left(\widetilde{u}_{0}^{2}+2 \widetilde{V}\right)^{1 / 2}$ according to the first half of Eq. (8). Equations (10) and (11) then yield $\widetilde{J}_{c} \rightarrow 6 / \widetilde{T}^{3}$ and $\widetilde{u}_{0}+\tilde{u}_{f} \rightarrow 3 / \widetilde{T}$, which may be combined to obtain the Child-Langmuir limiting current $J_{\mathrm{CL}}$ upon eliminating $\widetilde{T}$ :

$$
\tilde{J}_{\mathrm{CL}}=\frac{2}{9}\left[\tilde{u}_{0}+\left(\tilde{u}_{0}^{2}+2 \tilde{V}\right)^{1 / 2}\right]^{3} \text {. }
$$

For nonzero values of $\Omega$, Eqs. (10) and (11) give the relation between the limiting current $\left(\widetilde{J}_{c}\right)$ and the gap voltage (through $\widetilde{u}_{f}$ ) with $\widetilde{T}$ serving as a running parameter. The Child-Langmuir Law is recovered as $T \rightarrow 0$, as we have just shown, and the Hull cutoff condition is reached as $\widetilde{T}$ is increased to the value at which the right-hand side of Eq. (11) approaches zero [i.e., $\tilde{u}_{f} \rightarrow 0$ ].

To see clearly the effect of the transverse magnetic field $B$ on the limiting current, consider first the case $u_{0}=0$. In this case, the classical Child-Langmuir current becomes $\widetilde{J}_{\mathrm{CL}}=(2 / 9)(2 \widetilde{V})^{3 / 2}$ and Eqs. (8) and (9) give $\widetilde{u}_{f}=(2 \widetilde{V}-1)^{1 / 2}$, and $\widetilde{V}=\left(B_{\mathrm{H}} / B\right)^{2} / 2$. These relations then yield the limiting current as a function of magnetic field, as shown in the curve labeled $u_{0}=0$ in Fig. 1(a). The diode is
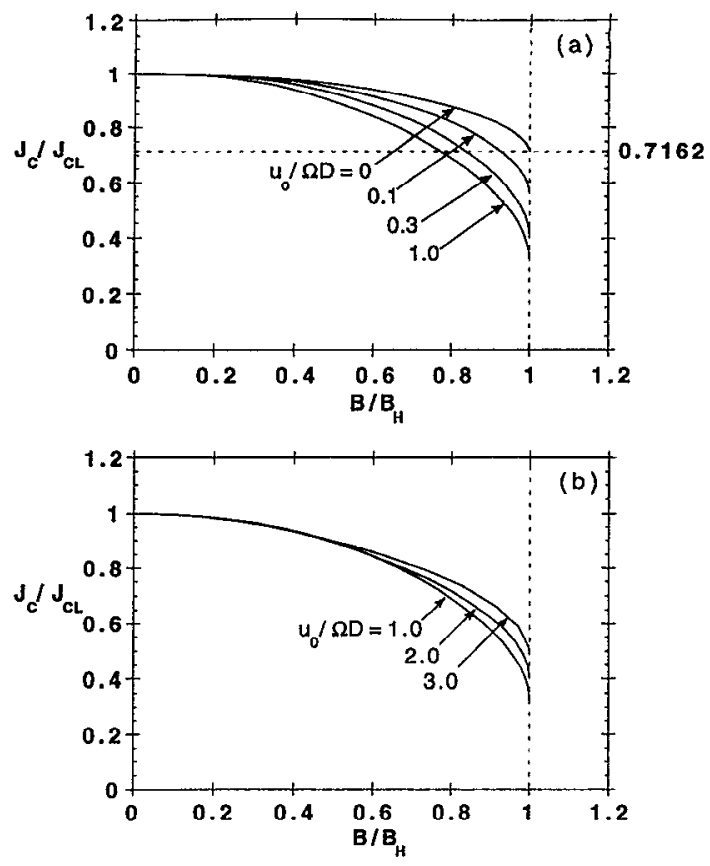

FIG. 1. The limiting current $\widetilde{J}_{\mathrm{C}}$, in units of the Child-Langmuir value $\widetilde{J}_{\mathrm{CL}}$, as a function of the magnetic field $B$, in units of the Hull cutoff value $B_{\mathrm{H}}$.

able to transmit the fraction $9 / 4 \pi=71.6 \%$ of the classical Child-Langmuir current ${ }^{12}$ as $B$ approaches $B_{\mathrm{II}}$ in this $u_{0}=0$ case $^{10}$

Alternatively, we may show from Eqs. (10) and (11) that the $u_{0}=0$ curve in Fig. 1 (a) is given by

$$
\frac{\widetilde{J}_{c}}{\widetilde{J}_{C L}}=\frac{9}{4} \frac{y^{2}}{\left(1+y^{2}\right)^{3 / 2}[h(y)]^{2}},
$$

where

$$
y=\frac{B / B_{\mathrm{H}}}{\sqrt{1-\left(B / B_{\mathrm{H}}\right)^{2}}}
$$

and $h(y)$ is shown in Fig. 2. Asymptotically, $h(y) \approx 3 y / 2$

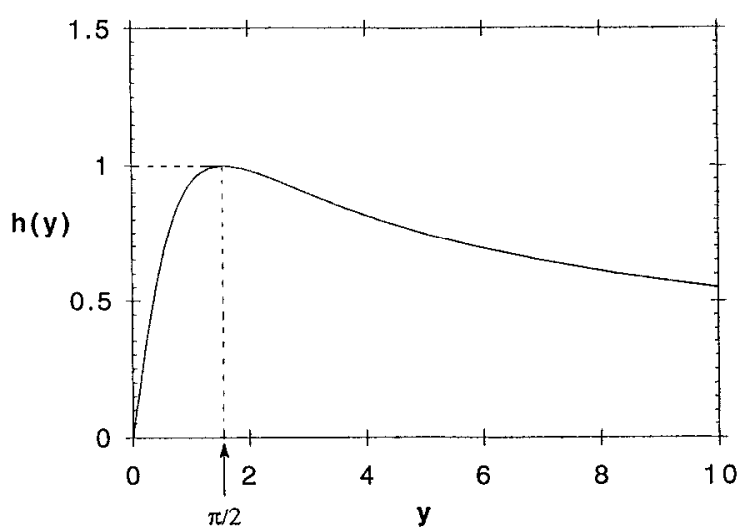

FIG. 2. The function $h(y)$. Asymptotically, $h(y) \rightarrow 3 y / 2$ for $y \ll 1$, and $h(y) \rightarrow \sqrt{\pi / y}$ for $y \gg 1$. 
for $y \ll 1$ and $h(y) \approx \sqrt{\pi / y}$ for $y \gg 1$. The function $x=h(y)$ is the inverse of the function $y=f(x)$, where

$y=f(x)= \begin{cases}\frac{1}{x^{2}}\left[\sin ^{-1}(x)-x \sqrt{1-x^{2}}\right], & 0<y<\frac{\pi}{2}, \\ \frac{1}{x^{2}}\left(\frac{\pi}{2}+\cos ^{-1}(x)+x \sqrt{1-x^{2}}\right), & \frac{\pi}{2}<y<\infty .\end{cases}$

In Eq. (15), $x$ ranges between zero and one and the principal values are to be used in the multivalued functions.

Equation (13) may be generalized to nonzero values of $\widetilde{u}_{0}$. In this case,

$$
\tilde{J}_{c}=1 / 2 y_{1}\left[h\left(y_{1}\right)\right]^{2}
$$

where

$$
y_{1}=1 /\left[\tilde{u}_{0}+\sqrt{\left(B_{\mathrm{H}} / B\right)^{2}-1}\right]
$$

and the function $h(y)$ is the same as that given in Fig. 2 . The ratio $\vec{J}_{C} / \vec{J}_{C L}$ may then be constructed upon using Eqs. (12) and (16). The results are shown in Fig. 1 (a) for $\widetilde{u}_{0} \leqslant 1$ and in Fig. 1(b) for $\tilde{u}_{0} \geqslant 1$. We have checked that these curves are reproduced by using Eqs. (10) and (11) with $\tilde{T}$ serving as a running parameter.

From Fig. 1, we see that the transverse magnetic field $B$ has only a very weak effect on the transmittable current, for all values of $B$ up to $B_{\mathrm{H}}$, in particular in the physically significant case $u_{0}=0$. As $B$ exceeds $B_{\mathrm{H}}$, steady current transmission to the anode is impossible, as expected [cf. Eq. (8)]; oscillatory transmission may occur, as in the unmagnetized diode for $J>J_{c}$.

\section{REMARKS}

The curves shown in Fig. 1 all have an infinite slope as $B \rightarrow B_{\mathrm{H}}$. This statement is proved in the Appendix. The implication is that a little adjustment of the magnetic field would cause a large jump in the transmittable current when $B$ is in the immediate vicinity of $B_{\mathrm{H}}$. This observation ties the limiting current to the Hull cutoff condition, which itself does not depend on the electron density [cf. Eq. (1)]. Indeed, the onset of a new state for $B>B_{\mathrm{H}}$ has already been strongly hinted by the theory, experiment, and particle simulation done by Pollack. ${ }^{10}$ In contrast to an unmagnetized diode, which is known to possess two critical currents (one physical and the other not realizable; see p. 72 of Ref. 8, for example), the properties of such critical currents for the case of a magnetized diode have not been adequately studied; but we conjecture that the one presented in this paper is the physically realizable branch. The stability of the crossed-field diode is an interesting subject but is beyond the scope of this paper; i.e., there is a need for numerical simulation.

The result that the limiting current, in the physically significant case $u_{0} \approx 0$, is insensitive to the magnetic field (up to $B_{\mathrm{H}}$ ) does not imply that the dynamic behavior of a high current crossed-field gap is necessarily similar to an ordinary gap (one without a transverse magnetic field). For example, in a crossed-field gap, the $x$-component velocity of an electron could be substantially reduced because of the presence of the transverse magnetic field, the instantaneous power transfer to that electron by the $x$ component of the electric field would then be significantly different from that in an ordinary gap. This, of course, is merely a reiteration of the well-known fact ${ }^{8}$ that crossed-field devices are markedly different from "O-type" devices. The latter include the traveling wave tube, gyrotron, klystron, free-electron laser, virtual cathode oscillator, etc., which do not possess a transverse magnetic field.

To summarize this paper, we have presented an analytic theory which bridges the gap between the classical Child-Langmuir law and the Hull cutoff condition. We have found that the limiting current is not significantly affected by the magnetic field, $B$, as long as $B$ is below the Hull cutoff value, if the electrons are released into the gap with negligible initial velocity.

\section{ACKNOWLEDGMENTS}

We thank Norm Dionne of Raytheon and Hunter McDowell of Varian for many useful discussions. We are grateful to the reviewer for noting the implications of the infinite slopes in Fig. 1 and adding several references.

This work was supported by the Naval Research Laboratory and by the Office of Naval Research.

\section{APPENDIX: PROOF THAT $\partial J_{G} / \partial B=\infty$ AS $B \rightarrow B_{H}$}

There are several ways to prove that the curves in Fig. 1 have an infinite slope as $B \rightarrow B_{\mathrm{H}}$. For the $u_{0}=0$ curve in Fig. 1(a), we may use the asymptotic expression

$$
h(y)=\sqrt{\frac{\pi}{y}}\left(1-\sqrt{\frac{1}{\pi y}}\right), \quad y \rightarrow \infty
$$

in Eq. (13). It is then straightforward to show from Eq. (13) that $\partial J_{\mathcal{c}} \partial B=\infty$ as $y \rightarrow \infty$, i.e., as $B \rightarrow B_{\mathrm{H}}$ by Eq. (14). Equation (A1) is easily established from Eq. (15).

For general values of $u_{0}$, we may use Eqs. (10) and (11) to write

$$
\frac{\partial \tilde{J}_{c}}{\partial B}=\left(\frac{\partial \tilde{J}_{c} / \partial \widetilde{T}}{\partial \tilde{u}_{f} / \partial \tilde{T}}\right) \cdot\left(\frac{\partial \tilde{u}_{f}}{\partial B}\right) .
$$

The last set of parentheses tends to infinity as $B \rightarrow B_{\mathrm{H}}$ by virtue of Eq. (8). The first set of parentheses is nonzero, according to Eqs. (10) and (11). This completes the proof.

'C. D. Child, Phys. Rev. 32, 492 (1911); I. Langmuir, ibid. 21, 419 (1923); G. Jaffe, ibid. 65, 91 (1944).

${ }^{2}$ See, e.g., R. C. Davidson, Physics of Nonneutral Plasmas (AddisonWesley, Redwood City, CA, 1990); S. Humphries, Charged Particle Beams (Wiley, New York, 1990); J. D. Lawson, Physics of Charged Particle Beams (Oxford University Press, Oxford, 1988); R. H. Miller, Intense Charged Particle Beams (Plenum, New York, 1982).

${ }^{3}$ H. Jory and A. Trivelpiece, J. Appl. Phys. 40, 3924 (1969).

${ }^{4}$ D. G. Colombant and Y. Y. Lau, Phys. Rev. Lett. 64, 2320 (1990), and references therein.

${ }^{5}$ Y. Y. Lau, D. Chernin, D. G. Colombant, and P. T. Ho, Phys. Rev. Lett. 66, 1446 (1991).

${ }^{6}$ A. W. Hull, Phys. Rev. 18, 31 (1921).

'T. J. Orzechowski and G. Bekefi, Phys. Fluids 22, 978 (1979).

${ }^{8} \mathrm{C}$. K. Birdsall and W. B. Bridges, Electron Dynamics of Diode Regions (Academic, New York, 1966), Chap. 5.

'J. C. Slater, Microwave Electronics (Van Nostrand, New York, 1950); 
E. Okress, Crossed-Field Microwave Devices (Academic, New York, 1961).

${ }^{10}$ M. A. Pollack, Univcrsity of California, Berkeley, California, Series No. 60 , Issue No. 485 (unpublished, 1962). The referee has kindly pointed out to us that this unpublished report of Pollack contains a wealth of information. Specifically, the $u_{0}=0$ curve in Fig. 1 (a) of the present paper is identical to Fig. III.4-1 of Pollack, which was reproduced as Fig. 5.05d of Ref. 8. While neither Pollack nor Birdsall showed infinite slope of the maximum transmittable current $\left(J_{c}\right)$ as $B / B_{\mathrm{H}} \rightarrow 1$, they did note the infinite slopes in the other variables such as the transit angle, transit time and $u$, as $B / B_{\mathrm{H}} \rightarrow 1$ in the $u_{0}=0$ case. (See Figs. 5.05a, b, c of Ref. 8, reproduced from Pollack.) Pollack's particle simulation and experiment showed that the anode noise current increased markedly as $B \rightarrow B_{\mathrm{H}}$, with noise currents far above full shot noise observed for
$B>B_{\mathrm{H}}$. A condensed version was given in M. A. Pollack and J. R. Whinnery, IEEE Trans. Electron Devices, ED-11, 81 (1964). Though highly suggestive, none of these earlier works made the claim that associated the limiting current with the Hull cutoff since they did not prove the "running out of solutions" for $J_{c}$ as $B \rightarrow B_{\mathrm{H}}$. The latter is accomplished in the Appendix of this paper.

${ }^{11}$ T. Van Duzer, IEEE Trans. Electron Devices ED-8, 78 (1961); ED-10, 370 (1963).

${ }^{12}$ This fraction $(9 / 4 \pi)$ was also noted by Slater on p. 344 of Ref. 9. It is also implicit in Fig. 5.05d of Ref. 8. This fraction of the ChildLangmuir current has also been known as the characteristic current by G. B. Collins, Microwave Magnetrons (McGraw-Hill, New York, 1948) 